\title{
A!
}

This is an electronic reprint of the original article.

This reprint may differ from the original in pagination and typographic detail.

Bruno, Elena; Mirabella, Salvo; Priolo, Francesco; Kuitunen, Katja; Tuomisto, Filip; Slotte, Jonatan; Giannazzo, Filippo; Bongiorno, Corrado; Raineri, Vito; Napolitani, Enrico

He implantation to control B diffusion in crystalline and preamorphized Si

Published in:

Journal of Vacuum Science and Technology. Part B.

DOI:

$10.1116 / 1.2816927$

Published: 01/01/2008

Document Version

Publisher's PDF, also known as Version of record

Please cite the original version:

Bruno, E., Mirabella, S., Priolo, F., Kuitunen, K., Tuomisto, F., Slotte, J., Giannazzo, F., Bongiorno, C., Raineri, V., \& Napolitani, E. (2008). He implantation to control B diffusion in crystalline and preamorphized Si. Journal of Vacuum Science and Technology. Part B., 26(1), 386-390. https://doi.org/10.1116/1.2816927

This material is protected by copyright and other intellectual property rights, and duplication or sale of all or part of any of the repository collections is not permitted, except that material may be duplicated by you for your research use or educational purposes in electronic or print form. You must obtain permission for any other use. Electronic or print copies may not be offered, whether for sale or otherwise to anyone who is not an authorised user. 


\section{He implantation to control B diffusion in crystalline and preamorphized Si}

E. Bruno, S. Mirabella, and F. PrioloK. Kuitunen, F. Tuomisto, and J. SlotteF. Giannazzo, C. Bongiorno, and V. RaineriE. Napolitani

Citation: Journal of Vacuum Science \& Technology B: Microelectronics and Nanometer Structures Processing, Measurement, and Phenomena 26, 386 (2008); doi: 10.1116/1.2816927

View online: http://dx.doi.org/10.1116/1.2816927

View Table of Contents: http://avs.scitation.org/toc/jvn/26/1

Published by the American Institute of Physics 


\title{
He implantation to control B diffusion in crystalline and preamorphized Si
}

\author{
E. Bruno, ${ }^{\text {a) }}$ S. Mirabella, and F. Priolo \\ MATIS CNR-INFM and Dipartimento di Fisica e Astronomia, Università di Catania, Via S. Sofia, I-95123 \\ Catania, Italy \\ K. Kuitunen, F. Tuomisto, and J. Slotte \\ Laboratory of Physics, Helsinki University of Technology, P.O. Box 1100, \\ FIN-02015 TKK Helsinki, Finland \\ F. Giannazzo, C. Bongiorno, and V. Raineri \\ CNR-IMM, Sezione di Catania, Stradale Primosole 50, I-95121 Catania, Italy \\ E. Napolitani \\ MATIS CNR-INFM and Dipartimento di Fisica, Università di Padova, Via Marzolo, 8, I-35131 Padova, \\ Italy
}

(Received 31 May 2007; accepted 30 October 2007; published 31 January 2008)

\begin{abstract}
We demonstrate that $\mathrm{He}$ can be a powerful tool to control B diffusion both in crystalline $(c-\mathrm{Si})$ and preamorphized $\mathrm{Si}(\mathrm{PA}-\mathrm{Si}$ ). By means of positron annihilation spectroscopy (PAS), we showed in He-implanted $c$-Si the formation after annealing of large open-volume defects at the implant projected range $R_{p}$ of $\mathrm{He}$ (voids) and of smaller vacancy-type defects toward the surface (nanovoids). In particular, these nanovoids locally suppress the amount of self-interstitials (Is) generated by B implantation, as verified by PAS, eventually reducing B diffusion and leading to a boxlike shape of the B-implanted profile. On the other hand, for B implantation in PA-Si, the authors demonstrated that if He-induced voids are formed between the end-of-range (EOR) defects and the surface, they act as a diffusion barrier for Is coming from the EOR defects. Indeed, this barrier strongly reduces diffusion of B placed in proximity of the surface. (C) 2008 American Vacuum Society. [DOI: 10.1116/1.2816927]
\end{abstract}

\section{INTRODUCTION}

Boron implantation in silicon is the most used method to selectively produce $p$-doped regions in silicon. It is also well asserted the need to progressively increase the local B concentration in order to obtain higher and higher electrical activation levels, while greatly reducing the junction depth. Many methodologies have been proposed to face this problem by using implantation of $\mathrm{B}$ in crystalline $(c-\mathrm{Si})$ or preamorphized (PA-Si) silicon. Both approaches present advantages and disadvantages regarding the impact of the implantation damage on B diffusion and electrical activation. When implanted directly in $c$-Si, B interacts with selfinterstitials Is produced by the implant itself, thus suffering the well-known transient-enhanced diffusion ${ }^{1,2}$ and the agglomeration in B-I complexes (BICs) that make it electrically inactive. ${ }^{1,3-5} \mathrm{~B}$ implantation in PA-Si overcomes these effects, because after regrowth [solid-phase epitaxy (SPE)] of the amorphous $\mathrm{Si}(a-\mathrm{Si})$ layer, B does not overlap to any interstitial. Furthermore, its activation level reaches values $\left(3.5 \times 10^{20} \mathrm{~B} / \mathrm{cm}^{3}\right)($ Ref. 6) significantly higher than those obtained in $c$-Si. Moreover, the implanted B profile is shallower than that obtainable in $c$-Si because channeling effects are absent in $a$-Si. Nonetheless, the end-of-range (EOR) defects, left at the original amorphous-crystal $(a-c)$ interface after SPE, dissolve under further annealing. This produces a

${ }^{a)}$ Electronic mail: elena.bruno@ct.infn.it flux of Is toward the surface that interacts with the implanted $\mathrm{B}$ and induces its diffusion and deactivation, deteriorating the junction formed. ${ }^{7-9}$

Nonequilibrium B diffusion and BIC formation have been the subject of many studies to control and suppress them. Different approaches have been proposed, ${ }^{10-14}$ but a method suitable for both $c$-Si and PA-Si, that is also easily implemented in industrial Si-based device production, is still lacking.

A possible solution could be $\mathrm{He}$ implantation in order to selectively and locally create sinks for Is. In fact, when implanted at high doses in $\mathrm{Si}, \mathrm{He}$ is able to produce a local supersaturation of vacancies (Vs) that can evolve in empty voids under annealing ${ }^{15,16}$ and act as a sink for Is. ${ }^{17}$ In detail, a deep void layer forms at the He projected range $\left(R_{p}\right)$, while smaller nanovoids form between the surface and the deep void layer itself, as we previously showed. ${ }^{18-20}$ In particular, we recently demonstrated that $\mathrm{He}$ implantation allows to control and even engineer the implanted B profile. ${ }^{18-20}$ In fact, the He-induced damage positively affects implanted B by reducing its diffusion and leading to a boxlike shape of the B profile. ${ }^{18-20}$

In this article, we report a study on He implantation to control B diffusion either in $c$-Si or in PA-Si, critically reviewing the mechanisms and the possible advantages. For $c$-Si, by means of positron annihilation spectroscopy (PAS), we definitely demonstrated that the defects near the surface affecting the diffusion of implanted B are vacancy-type defects that appear to be small, but larger than divacancies. 
These defects produce the annihilation of the implantationrelated damage, thus preventing Is from interacting with $B$ and reducing the above-mentioned detrimental phenomena. On the other hand, for B implantation in PA-Si, we demonstrated that, if He-induced voids are formed between the EOR defects and the surface, they act as a filter for Is coming from the EOR defects, preventing any diffusion of B placed in proximity of the surface.

\section{EXPERIMENT}

The study in $c$-Si was performed partially on a $2-\mu \mathrm{m}$-thick crystalline Si film grown by molecular-beam epitaxy (MBE) on a 5 in., $(2 \times 1)$ reconstructed, (100)-oriented $n$-type $\mathrm{Si}$ wafer containing an array of 190-nm-spaced boron delta doping $(\delta)$ with a peak concentration of about $2 \times 10^{18} \mathrm{~B} / \mathrm{cm}^{3}$, and partially on $\mathrm{Si}(100)$ czochralski $(\mathrm{Cz})$ wafers, $n$-type, $1.5-4 \Omega \mathrm{cm}$. Part of the samples were implanted with $80 \mathrm{keV} \quad \mathrm{He}$ ions at $3 \times 10^{16}$ or $8 \times 10^{16} \mathrm{ions} / \mathrm{cm}^{2}\left(R_{p} \sim 600 \mathrm{~nm}\right)$. B implantation $(12 \mathrm{keV}$, $5 \times 10^{14}$ ions $\left./ \mathrm{cm}^{2}, R_{p} \sim 40 \mathrm{~nm}\right)$ was also performed on part of the $\mathrm{Cz}$ samples, some of which coimplanted with He. All implanted samples were first annealed at $800{ }^{\circ} \mathrm{C}$ for $10 \mathrm{~min}$ to form $\mathrm{He}$ induced voids and nanovoids, and then for $100 \mathrm{~min}$ at the same temperature.

The experimental study in PA-Si was carried out on a $0.80-\mu$ m-thick epitaxial Si film grown on a 6 in., $(2 \times 1)$ reconstructed, (100)-oriented $p^{+}$-type $\mathrm{Si}$ wafer by MBE, containing a boron delta layer $(\delta)$ at $\sim 100 \mathrm{~nm}$ from the surface, having a peak concentration of about $5 \times 10^{17} \mathrm{~B} / \mathrm{cm}^{3}$. The sample was then amorphized from the surface to a depth of $\sim 510 \mathrm{~nm}$ by implanting $\mathrm{Si}^{-}$ions $\left(3 \times 10^{15}\right.$ ions $/ \mathrm{cm}^{2}$ at $250 \mathrm{keV}$, plus $2 \times 10^{15}$ ions $/ \mathrm{cm}^{2}$ at $40 \mathrm{keV}$ ) at the liquidnitrogen temperature. The amorphized sample was regrown at $550{ }^{\circ} \mathrm{C}$ for $100 \mathrm{~min}$. After SPE, part of the samples are implanted with $40 \mathrm{keV} \mathrm{He}$ ions at $5 \times 10^{16}$ ions $/ \mathrm{cm}^{2}$ $\left(R_{p} \sim 390 \mathrm{~nm}\right)$. Finally, all samples were annealed at $850{ }^{\circ} \mathrm{C}$ for $20 \mathrm{~min}$ to either form He-induced voids (between 290 and $410 \mathrm{~nm}$ ) or let the EOR defects (at about $510 \mathrm{~nm}$ ) evolve and dissolve by emitting Is. Some pieces of the asgrown crystalline sample were also processed at $850{ }^{\circ} \mathrm{C}$ $20 \mathrm{~min}$ as a reference.

Thermal annealing up to 20 min were performed with rapid thermal annealing, whereas prolonged annealing was performed in a conventional furnace. All annealings were performed in a controlled $\mathrm{N}_{2}$ atmosphere. B chemical profiling was obtained by secondary ion mass spectrometry analyses, performed with a CAMECA IMS- 4 instrument $(3 \mathrm{keV}$ $\mathrm{O}_{2}^{+}$analyzing beam). PAS was performed by using a lowenergy positron beam to detect the Doppler shift in the $511 \mathrm{keV}$ annihilation line. The shape of the annihilation peak was described using the conventional shape parameter $S$ corresponding to the fraction of positrons annihilated with the low-momentum (valence) electrons. Thus, an increase in the $S$ parameter from the bulk value is a signal of vacancies in the material. A more thorough explanation of the measurement method can be found in Ref. 21. Because the native oxide at the surface of the samples disturbs the measurement

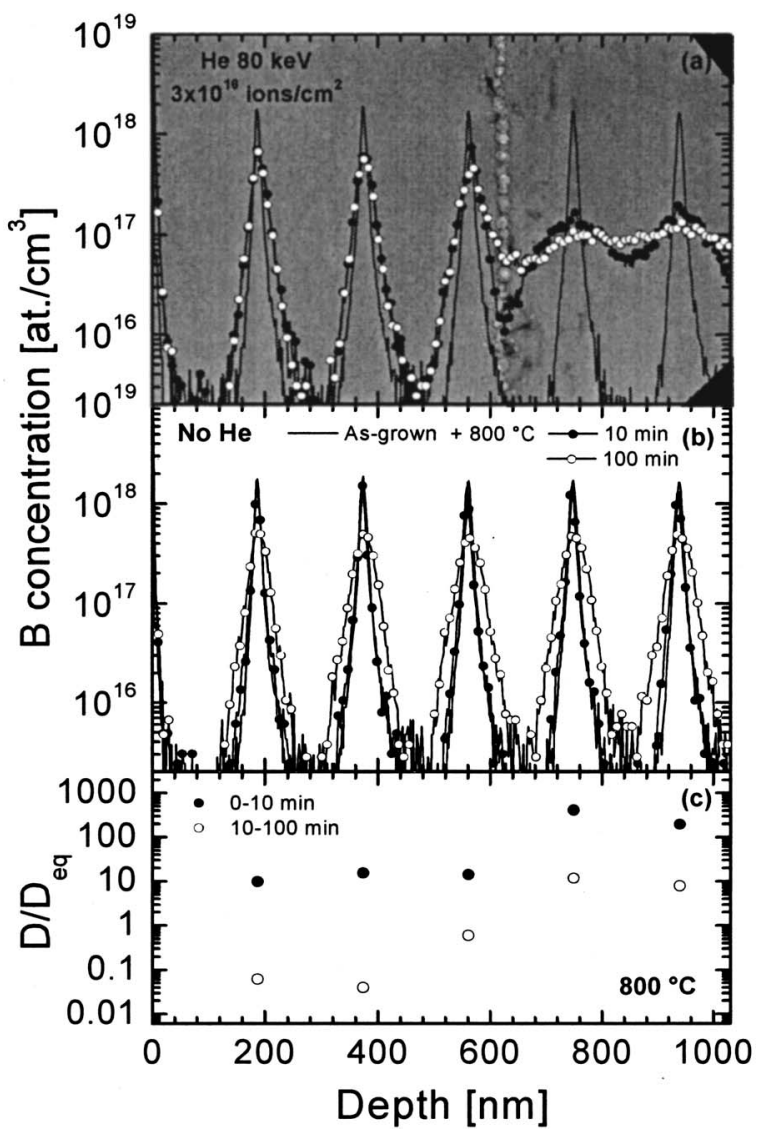

FIG. 1. Chemical B profiles after MBE growth (continuous lines) and after thermal annealing at $800{ }^{\circ} \mathrm{C}$ for $10 \mathrm{~min}$ (line plus closed circles) and $100 \mathrm{~min}$ (line plus open circles) in a sample implanted with $80 \mathrm{keV} \mathrm{He}, 3$ $\times 10^{16}$ ions $/ \mathrm{cm}^{2}$ (a) and in a He-free sample (b). The XTEM image of the He-implanted sample after $800{ }^{\circ} \mathrm{C}$ for $10 \mathrm{~min}$ is also reported as a background of panel (a). Panel (c) shows the enhancement of B diffusivity at the B deltas with respect to the equilibrium one, where $D_{\text {eq }}$ is calculated in the He-free sample.

data (see Ref. 22), the samples were etched in HF acid before the measurements. Cross-section transmission electron microscopy (XTEM) images were performed with a $200 \mathrm{kV}$ Jeol JEM 2010 microscope, in such a way to enhance features of the cavities, linear defects such as $\{311\}$ or dislocation loops.

\section{RESULTS AND DISCUSSION}

In the present section, we report results on the use of $\mathrm{He}$ implantation to produce $p$-type junctions of B in Si. We first studied the defect distribution created by $\mathrm{He}$ implantation through a $c$-Si sample. Then, we properly used the different regions of this defect distribution to control $\mathrm{B}$ diffusion both in $c$-Si, where $\mathrm{B}$ is introduced by implantation, and in PA-Si, where $\mathrm{B}$ is introduced by MBE.

\section{Distribution of defects produced by He implantation in $\mathrm{Si}$}

Figure 1 shows the B concentration profiles in the presence of a He implant, from which the point-defect distribution can be inferred. In detail, Figs. 1(a) and 1(b) show the 
chemical profiles of five $\mathrm{B}$ delta layers grown by MBE in He-implanted $\left(80 \mathrm{keV}, 3 \times 10^{16}\right.$ ions $\left./ \mathrm{cm}^{2}\right)$ and He-free samples, respectively. In particular, the as-grown profile (continuous lines) and those after thermal annealing at $800{ }^{\circ} \mathrm{C}$ for $10 \mathrm{~min}$ (lines plus closed circles) and $100 \mathrm{~min}$ (lines plus open circles) are shown in both cases. The XTEM image of the He-implanted sample after $800{ }^{\circ} \mathrm{C}$ for $10 \mathrm{~min}$ is also reported as a background of Fig. 1(a), showing the presence of a layer of He-induced cavities at $\sim 600 \mathrm{~nm}$.

By comparing Figs. 1(a) and 1(b), it is clear that He implantation induces a nonuniform delta broadening, which means a nonuniform distribution of point defects through the sample, as reported in Fig. 1(c). Figure 1(c) shows the enhancement of $\mathrm{B}$ diffusivity at the $\mathrm{B}$ deltas with respect to the equilibrium diffusivity, where $D_{\text {eq }}$ is calculated in the Hefree sample. After the shorter annealing, the two delta layers at $\sim 750$ and $\sim 950 \mathrm{~nm}$ beyond the deep void layer at the $\mathrm{He}$ $R_{p}$ undergo a greatly enhanced diffusion with respect to equilibrium. On the other hand, the shallower deltas undergo smaller diffusion (though still higher than equilibrium). After the prolonged annealing at $800{ }^{\circ} \mathrm{C}$ for $100 \mathrm{~min}$ the situation changes. In the case of the He-implanted sample [Fig. 1(a)], the two deeper delta layers continue to strongly broaden, whereas the shallower layers, unlike before, diffuse with a B diffusivity much lower than the equilibrium one. On the other hand, in the He-free sample, all the B delta layers thermally diffuse in the same way, emphasizing that the I distribution is uniform and equal to the equilibrium one throughout all the sample. It is worth noting that in the He-implanted sample, the lowering of the middle B delta layer at $\sim 560 \mathrm{~nm}$ could be related to the closeness of the void layer at $R_{p}{ }^{23}$

The effect can be explained as described in the following. In the case of the He-free sample, Is responsible for B diffusion are thermally generated because no point-defect source or sink is present in our sample, except the surface. This means that the I concentration is uniform and, as a consequence, the B delta layers must enlarge everywhere due to thermal diffusivity. In the sample implanted with $\mathrm{He}$, the situation is different. In fact, He implanted in $\mathrm{Si}$ is able to form $\mathrm{He}-\mathrm{V}$ complexes that allow vacancies produced by the implant itself to survive under annealing and to aggregate in larger V-clusters. ${ }^{16}$ In the meantime, Is generated along the He ion tracks annihilate elsewhere, while interacting with B before disappearing. Indeed, the diffusion of the shallower B deltas after $800{ }^{\circ} \mathrm{C}$ for $10 \mathrm{~min}$ probably occurs at the first stages of annealing or even during the He implant itself, when a moderate amount of Is are still present. For prolonged annealing, once implant-related Is are annihilated in the region between the surface and $R_{p}$, a supersaturation of open-volume defects persists that act as a sink for Is, including those thermally produced. As a consequence, B delta layers are prevented from interacting with Is and hence to diffuse. On the other hand, as far as the strong diffusion of the deeper deltas is concerned, it is related to the excess of Is, often aggregated in extended defects that form beyond the void layer at the $R_{p}$ of He.

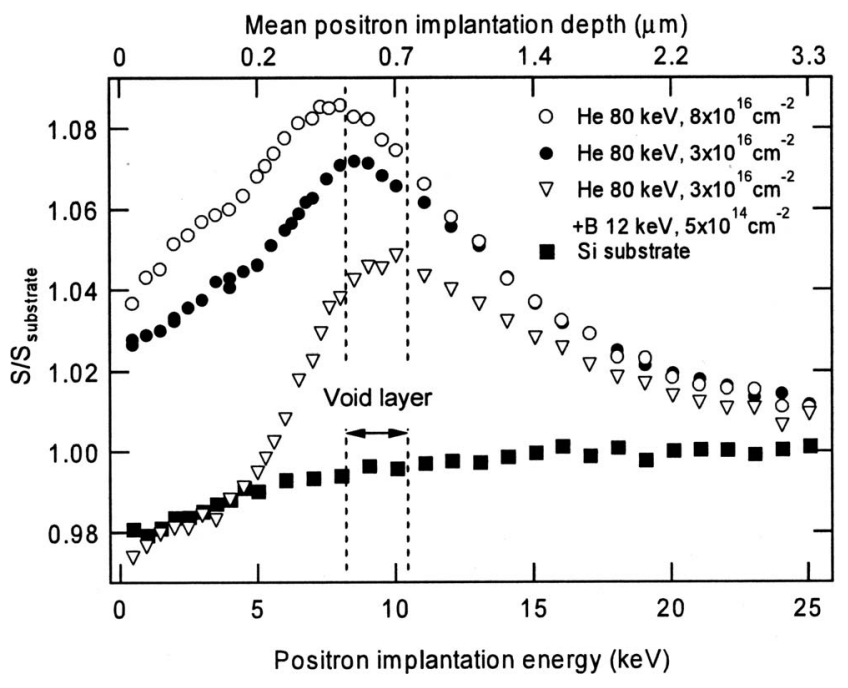

FIG. 2. The low-momentum parameter $S$ as a function of positron implantation energy in samples implanted only with $80 \mathrm{keV} \mathrm{He}$ with doses of $3 \times 10^{16}$ (closed circles) and $8 \times 10^{16}$ (open circles) ions $/ \mathrm{cm}^{2}$ and subjected to annealing at $800{ }^{\circ} \mathrm{C}$ for $10 \mathrm{~min}$, as before. The $S$ parameter has been normalized to the value in the substrate far from the surface (closed squares, $S_{\text {substrate }}=0.556$ ), and the mean positron implantation depth has also been included in the figure (top scale). The $S$ parameter in a sample implanted both with $\mathrm{He}$ and with $\mathrm{B}\left(80 \mathrm{keV} \mathrm{He}, 3 \times 10^{16}\right.$ ions $/ \mathrm{cm}^{2}$ and $12 \mathrm{keV} \mathrm{B}$, $5 \times 10^{14} \mathrm{~B}$ ions $/ \mathrm{cm}^{2}$ ) is also shown (open down triangles).

Summarizing, a He implant produces an I-rich region beyond its $R_{p}$ and a vacancy-rich region between $R_{p}$ and the surface. In particular, the $\mathrm{V}$-rich region can be divided into the area at $R_{p}$, where a layer of large cavities forms, and a shallower region toward the surface with much smaller V-type defects eventually organized in more complex V clusters or nanovoids. The above distribution can be properly exploited to control point defects in Si samples and, hence, B diffusion both in $c$-Si and in $\mathrm{PA}-\mathrm{Si}$, as it will be reported in the following sections.

\section{He implantation in c-Si: Shallow nanovoids effects on implanted B}

In this section, we study how the $\mathrm{V}$ distribution produced by $\mathrm{He}$ implantation changes under further shallow implant, and consequently how it can affect the diffusion of implanted B $\left(12 \mathrm{keV}, 5 \times 10^{14} \mathrm{~B}\right.$ ions $\left./ \mathrm{cm}^{2}\right)$. We previously demonstrated that the $\mathrm{He}$ induced damage reduces $\mathrm{B}$ diffusion. ${ }^{18}$ Moreover, the B profile after annealing becomes progressively narrower, steeper, and higher, with a shape more and more similar to a box, as the He-implanted dose increases. $^{19,20}$

Figure 2 shows the $S$ parameter as a function of positron implantation energy in samples implanted only with $80 \mathrm{keV}$ He with doses of $3 \times 10^{16}$ (closed circles) and 8 $\times 10^{16}$ ions $/ \mathrm{cm}^{2}$ (open circles) and subjected to annealing at $800{ }^{\circ} \mathrm{C}$ for $10 \mathrm{~min}$, as before. The $S$ parameter has been scaled to the value in the substrate (closed squares, $S_{\text {substrate }}$ $=0.556$ ), and the mean positron implantation depth has also been included in the figure. The $S$ parameter as a function of positron implantation energy in a sample implanted both 
with $\mathrm{He}$ and with $\mathrm{B}\left(80 \mathrm{keV} \mathrm{He}, 3 \times 10^{16}\right.$ ions $/ \mathrm{cm}^{2}$, and $12 \mathrm{keV} \mathrm{B}, 5 \times 10^{14} \mathrm{~B}$ ions $/ \mathrm{cm}^{2}$ ) is also shown (open down triangles). A very high peak is visible at the positron implantation energies of $8-10 \mathrm{keV}$ for all three He-implanted samples (with and without B). Moreover, the two B-free samples implanted with He show a kink in the $S$ parameter curve and a slight plateau at about $3-5 \mathrm{keV}$. Indeed, the He-implanted sample further implanted with B shows a $S$ signal at 8-10 keV lower than the two B-free samples, whereas for lower positron energies (below $4 \mathrm{keV}$ ), $S$ closely follows the curve measured from the substrate.

PAS is a very suitable tool for detecting defects containing open volume. In particular, the increase in the $S$ parameter in the implanted samples is a clear signal of vacancies in the material. The highest peaks, at the positron implantation energies of $8-10 \mathrm{keV}$, are due to the deep voids formed at the $R_{p}$ of He implantation. However, the kink in the $S$ parameter curve and the hint of a plateau at about 3-5 keV between the surface and $R_{p}$ in the two samples without $\mathrm{B}$ implantation (open and closed circles) indicate that there exists a layer containing open-volume defects that differ from the voids at $R_{p}$. However, the value of the $S$ parameter at the kink indicates that these defects have a smaller open volume than the voids at $R_{p}$, but are larger than a divacancy. On the other hand, the superimposition of $S$ in the sample coimplanted with B and $\mathrm{He}$ (open down triangles) and the substrate (closed squares) for energies lower than $4 \mathrm{keV}$ indicates that nanovoids are absent in this region because the fraction of positrons trapped by the voids is greatly reduced.

Also, the height of the peak at $8-10 \mathrm{keV}$ is clearly lowered from the value measured from the sample without B implantation, as previously observed. This could mean either that the concentration of defects trapping positrons in correspondence of the void layer at the He $R_{p}$ is reduced or that the defects size is smaller. However, we cannot exclude that the $S$ signal at the deep void layer is modified by an interaction between positrons and shallow charged $\mathrm{B}$ ions.

Our previous XTEM analyses and $\mathrm{Cu}$ gettering experiments ${ }^{18-20}$ suggested that the region between the surface and the $R_{p}$ of He ions was rich in vacancies, possibly organized in clusters or nanovoids. Moreover, these V clusters were assumed to be responsible for modifying the diffusion, leading to a boxlike shape of the implanted B due to the consequent nonuniform I distribution present in the sample, and also for improving B electrical activation. ${ }^{18-20}$

In this section, we definitively demonstrated by PAS that He produces small $V$ clusters larger than divacancies toward the surface. The distribution of these $V$ clusters is then modified by B implantation. B implantation-related damage inhibits the formation of these V-related defects in the B-implanted region. Indeed, in the shallower region, V clusters that eventually formed disappear because of the B implant-related damage; thus, the I supersaturation is high and B is characterized by high diffusivity. On the other hand, at deeper depths, where nanovoids are still present, B diffusivity is very low because the I concentration is the equilib-

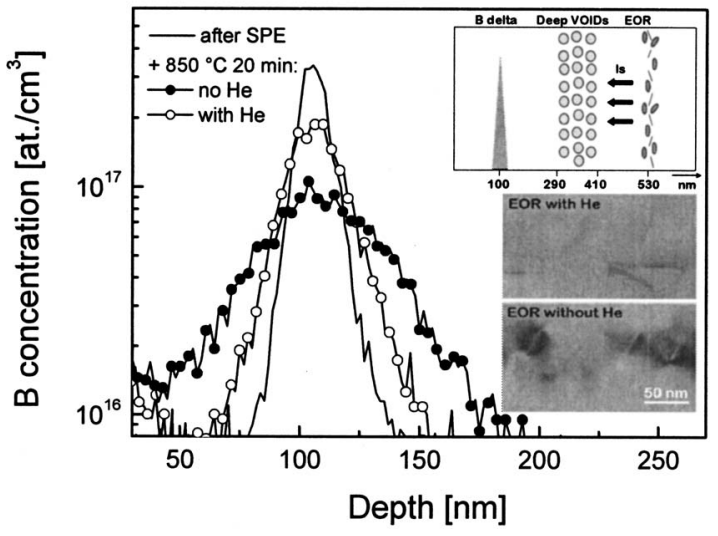

FIG. 3. Chemical profiles of a B delta grown by MBE after amorphization and SPE (continuous line) and after the further annealing at $850^{\circ} \mathrm{C}$ in a He-implanted $\left(40 \mathrm{keV}, 2 \times 10^{16}\right.$ ions $/ \mathrm{cm}^{2}$, open circles) sample and in a He-free sample (closed circles), respectively. The upper inset of the figure is a schematic of the experiment in PA-Si, while the lower one shows the EOR defects in the He-implanted and He-free samples.

rium value or even lower because of the nanovoid I trapping. As a consequence, B diffuses and accumulates here, progressively assuming a boxlike shape.

\section{He implantation in PA-Si: Voids as a barrier for EOR defects}

In this section, we focus on the large cavity layer formed at $R_{p}$ of the $\mathrm{He}$ implant, showing its potential in PA-Si samples. The first inset of Fig. 3 shows a schematic of the experiment. A sample containing a B delta near the surface, used as a marker for $I_{s}$, was grown by $\mathrm{MBE}$ and subsequently amorphized and regrown at low temperature $\left(550^{\circ} \mathrm{C}\right)$ by SPE, to leave I-type defects beyond the $a-c$ interface. After that, the sample was implanted with $\mathrm{He}$ in such a way that the He projected range, where the large cavity layer forms, was between the B delta and the original $a-c$ interface. Finally, the sample was annealed at high temperature to simultaneously form voids and make EOR defects evolve and eventually dissolve by emitting Is.

Figure 3 shows the chemical profiles of the B delta after SPE (continuous line) and after the further annealing at $850{ }^{\circ} \mathrm{C}$ for $20 \mathrm{~min}$ in a He-implanted $(40 \mathrm{keV}, 2$ $\times 10^{16}$ ions $/ \mathrm{cm}^{2}$, open circles) and in a He-free sample (closed circles), respectively. Looking at the B profiles, it is clear that the diffusion in the He-implanted sample is much lower than in the He-free sample. In particular, B diffusivity is $\sim 0.19 \mathrm{~nm}^{2} / \mathrm{s}$ in the He-free sample and becomes $\sim 0.04 \mathrm{~nm}^{2} / \mathrm{s}$ if He-induced voids are interposed between B and EOR defects, reducing $\mathrm{B}$ diffusivity by a factor of about 5. Moreover, the value of $0.04 \mathrm{~nm}^{2} / \mathrm{s}$ in the He-implanted sample agrees with the equilibrium diffusivity observed in the as-grown samples processed with the same annealing (not shown), indicating that the void layer acts as a perfect barrier for Is coming from the EOR defects.

The two lower insets of Fig. 3 show the XTEM images of the EOR defects (centered at $520 \mathrm{~nm}$ and whose band is $\sim 50 \mathrm{~nm}$ thick) in the He-implanted and He-free sample. We 
see that the EOR defects are very different in the two cases, although the process steps (amorphization implant, SPE, and further annealing at $850^{\circ} \mathrm{C}$ ) were exactly the same, except for the He implantation. In the He-free sample, XTEM analyses clearly reveal the presence of a band of dislocation loops just below the original $a-c$ interface, as shown by the presence of the coffee-bean contrast typical of these I-type defects. When the sample is implanted with He, the EOR defect morphology changes. Most of the EOR defects consist of linear defects, several hundreds of nanometers long, whereas almost no dislocation loops are present.

These results demonstrate that He-related damage can greatly affect the number of interstitial also in PA-Si. He produces a layer of voids at its $R_{p}$ that act as a sink for Is present beyond the near $a-c$ interface. As a consequence, EOR defects cannot coarse into large and stable dislocation loops, but only aggregate in elongated I clusters, such as $\{311\}$. Moreover, the void layer at $R_{p}$, in addition to inhibiting the loop formation, acts as an efficient barrier for those Is moving toward the surface that are emitted by the EOR band.

This phenomenon could be applied to the preamorphization methodology used to produce $\mathrm{B}$ junctions in $\mathrm{Si}$ by ion implantation. Once the amorphous layer has been regrown, the I flux coming from the EOR band under further annealing can be stopped by a void layer interposed between EOR defects and the surface, preventing the implanted B to interact with Is and diffuse.

\section{CONCLUSIONS}

We studied the effect of He implantation on B diffusion either in $c$-Si or in PA-Si, showing the role of nanovoids extending from $R_{p}$ up to the surface or of the deep void layer, respectively.

In detail, B diffusion in $c$-Si can be properly modified by means of $\mathrm{He}$ implantation. Indeed, surface small openvolume defects induced by He efficiently trap Is produced by B implantation, whereas they disappear by I annihilation. This leads to a peculiar B boxlike shape of the implanted B profile. On the other hand, when applied in PA-Si, He implantation can be used to prevent B placed near the surface to diffuse. In fact, the deep void layer, if properly formed between the original $a-c$ interface and the surface, can stop the I flux emitted by the EOR defects, acting as a diffusion barrier for Is. Thus, we can state that $\mathrm{He}$ is a very attractive instrument for controlling the point-defect distribution both in $c$-Si and PA-Si. The described mechanism should be taken into account if He implantation is proposed as a versatile and advantageous technique to control $\mathrm{B}$ diffusion in practical applications in Si-based microelectronics.

\section{ACKNOWLEDGMENTS}

The authors wish to thank C. Percolla and S. Tatì (MATIS CNR-INFM Catania, Italy), A. Marino (IMM-CNR Catania, Italy), and R. Storti (University of Padova, Italy) for their contributions.

${ }^{1}$ P. A. Stolk, J. H.-J. Gossmann, D. J. Eaglesham, D. C. Jacobson, C. S. Rafferty, G. H. Gilmer, M. Jaraiz, J. M. Poate, H. S. Luftman, and T. E. Haynes, J. Appl. Phys. 81, 6031 (1997), and references therein.

${ }^{2}$ S. C. Jain, W. Schoenmaker, R. Lindsay, P. A. Stolk, S. Decoutere, M. Willander, and H. E. Maes, J. Appl. Phys. 91, 8919 (2002), and references therein.

${ }^{3}$ L. Pelaz, M. Jaraiz, G. H. Gilmer, H.-J. Gossmann, C. S. Rafferty, D. J. Eaglesham, and J. M. Poate, Appl. Phys. Lett. 70, 17 (1997).

${ }^{4}$ S. Mirabella, E. Bruno, F. Priolo, D. De Salvador, E. Napolitani, A. V. Drigo, and A. Carnera, Appl. Phys. Lett. 83, 680 (2003).

${ }^{5}$ D. De Salvador, E. Napolitani, G. Bisognin, A. Carnera, E. Bruno, S. Mirabella, G. Impellizzeri, and F. Priolo, Appl. Phys. Lett. 87, 221902 (2005).

${ }^{6}$ S. Solmi, E. Landi, and F. Baruffaldi, J. Appl. Phys. 66, 3250 (1990).

${ }^{7}$ C. Bonafos, M. Omri, B. de Mauduit, G. BenAssayag, A. Claverie, D. Alquier, A. Martinez, and D. Mathiot, J. Appl. Phys. 82, 2855 (1997).

${ }^{8}$ L. S. Robertson, M. E. Law, K. S. Jones, L. M. Rubin, J. Jackson, P. Chi, and D. S. Simons, Appl. Phys. Lett. 75, 3844 (1999).

${ }^{9}$ B. J. Pawlak, R. Surdeanu, B. Colombeau, A. J. Smith, N. E. B. Cowern, R. Lindsay, W. Vandervorst, B. Brijs, O. Richard, and F. Cristiano, Appl. Phys. Lett. 84, 2055 (2004).

${ }^{10}$ A. Smith, N. E. B. Cowern, R. Gwilliam, B. J. Sealy, B. Colombeau, E. J. H. Collart, S. Gennaro, D. Giubertoni, M. Bersani, and M. Barozzi, Appl. Phys. Lett. 88, 082112 (2006).

${ }^{11}$ D. F. Downey, J. W. Chow, E. Ishida, and K. S. Jones, Appl. Phys. Lett. 73, 1263 (1998).

${ }^{12}$ G. Impellizzeri, J. H. R. dos Santos, S. Mirabella, F. Priolo, E. Napolitani, and A. Carnera, Appl. Phys. Lett. 84, 1862 (2004).

${ }^{13}$ E. Napolitani, A. Coati, D. De Salvador, A. Carnera, S. Mirabella, S. Scalese, and F. Priolo, Appl. Phys. Lett. 79, 4145 (2001).

${ }^{14}$ N. E. B. Cowern, A. Cacciato, J. S. Custer, F. W. Saris, and W. Vandervorst, Appl. Phys. Lett. 68, 1150 (1996).

${ }^{15}$ C. C. Griffioen, J. H. Evans, P. C. De Jong, and A. Van Veen, Nucl. Instrum. Methods Phys. Res. B 27, 417 (1987).

${ }^{16}$ V. Raineri, M. Saggio, and E. Rimini, J. Mater. Res. 15, 1449 (2000) and references therein.

${ }^{17}$ V. Raineri and S. U. Campisano, Appl. Phys. Lett. 69, 1783 (1996).

${ }^{18}$ S. Mirabella, E. Bruno, F. Priolo, F. Giannazzo, C. Bongiorno, V. Raineri, E. Napolitani, and A. Carnera, Appl. Phys. Lett. 88, 191910 (2006).

${ }^{19}$ E. Bruno, S. Mirabella, F. Priolo, E. Napolitani, C. Bongiorno, and V. Raineri, J. Appl. Phys. 101, 023515 (2007).

${ }^{20}$ E. Bruno, S. Mirabella, F. Priolo, E. Napolitani, F. Giannazzo, and V. Raineri, Nucl. Instrum. Methods Phys. Res. B 257, 181 (2007).

${ }^{21}$ K. Saarinen, P. Hautojärvi, and C. Corbel, in Identification of Defects in Semiconductors, edited by M. Stavola (Academic, New York, 1998), p. 209.

${ }^{22}$ M. Rummukainen, I. Makkonen, V. Ranki, M. J. Puska, K. Saarinen, and H.-J. K. Gossmann, Phys. Rev. Lett. 94, 165501 (2005).

${ }^{23}$ F. Roqueta, D. Alquier, L. Ventura, Ch. Dubois, and R. Jérisian, Nucl. Instrum. Methods Phys. Res. B 183, 318 (2001). 